\title{
Extratos de Piper marginatum e Azadirachta indica no controle de Colletotrichum scovillei em pimentão
}

\author{
Emmanuelle Rodrigues Araújo(1), Wolfgang Harand(2), Iwanne Coelho Lima(1), Fernanda Carolina Ribeiro Dias ${ }^{(3)}$, \\ Adelmo Adriane Duarte de Santana(1), Rejane Rodrigues da Costa e Carvalho(1) e Delson Laranjeira(1) \\ (1)Universidade Federal Rural de Pernambuco, Departamento de Agronomia, Área de Fitossanidade, Laboratório de Fungos de Solos, Rua \\ Dom Manoel de Medeiros, s/nº, Dois Irmãos, CEP 52171-900 Recife, PE, Brasil. E-mail: manucg@gmail.com, coelho.iwanne@yahoo.com.br, \\ adelmoadriane@ig.com.br, rejanercosta@yahoo.com.br, delson@depa.ufrpe.br (2)Centro de Tecnologias Estratégicas do Nordeste, Central \\ Analítica, Avenida Professor Luiz Freire, № 1, Cidade Universitária, CEP 50740-540 Recife, PE, Brasil. E-mail: wolfgang@harand.at \\ (3)Universidade Federal de Pernambuco, Departamento de Ciências Biológicas, Avenida Professor Morais Rego, no 1235, Cidade Universitária, \\ CEP 50670-420 Recife, PE, Brasil. E-mail: fernandaribeiro.dias@hotmail.com
}

Resumo - O objetivo deste trabalho foi avaliar o efeito de extratos de capeba (Piper marginatum) e nim (Azadirachta indica) sobre o fungo Colletotrichum scovillei e determinar o componente mais ativo no controle pós-colheita da antracnose em pimentão. A atividade de extratos metanólicos das folhas ( $P$. marginatum $\mathrm{e}$ $A$. indica) e das sementes (A. indica) a $0,125,250,500,1.000$ e 2.000 ppm, na inibição do crescimento micelial de C. scovillei in vitro foram avaliadas. O extrato metanólico de folhas de P. marginatum foi o mais ativo e, consequentemente, foi submetido ao fracionamento biomonitorado ("bioassay-guided fractionation"). Esse processo rendeu 10 frações majoritárias, obtidas por cromatografia líquida de alta performance, das quais a fração à concentração de 1,5 ppm inibiu o desenvolvimento de C. scovillei de forma mais eficiente do que o fungicida mancozeb.

Termos para indexação: Capsicum annuum, antracnose, controle alternativo, fracionamento biomonitorado, nim.

\section{Extracts of Piper marginatum and Azadirachta indica for the control of Colletotrichum scovillei in bell pepper}

\begin{abstract}
The objective of this work was to evaluate the effect of extracts of capeba (Piper marginatum) and neem (Azadirachta indica) on the fungus Colletotrichum scovillei, and evaluate the most active compound on the post-harvest control of anthracnose in bell pepper. Methanolic extracts activity of leaves ( $P$. marginatum and $A$. indica) and of seeds (A. indica) at $0,125,250,500,1,000$, and 2,000 ppm, for in vitro inhibition of mycelial growth of $C$. scovillei, were evaluated. The methanolic extract of $P$. marginatum leaves was the most active and, consequently, it was subjected to a bioassay-guided fractionation. This processing produced ten major compounds, obtained by preparative HPLC, from which the fraction at $1.5 \mathrm{ppm}$ concentration was capable of inhibiting mycelial growth and was more effective than the fungicide mancozeb.
\end{abstract}

Index terms: Capsicum annuиm, anthracnose, alternative control, bioassay-guided fractionation, neem.

\section{Introdução}

A antracnose é considerada uma das doenças pós-colheita mais importantes do pimentão (Capsicum annuum L.) (Kim et al., 2014). É ocasionada por fungos do gênero Colletotrichum, tais como C. gloeosporioides (Penz.) Penz. \& Sacc., C. capsici (Syd.) E. J. Butler \& Bisby, C. coccodes (Wallr.) S. Hughes, C. acutatum J. H. Simmonds (Azevedo et al., 2006) e C. scovillei Damm (Damm et al., 2012), que podem promover a doença desde a pré-colheita e estão associados a infecções que levam a grandes perdas pós-colheita de frutas e hortaliças (Azevedo et al., 2006).

O controle da antracnose pode ser realizado por meio de diversas medidas integradas, como a utilização de sementes sadias, a realização do plantio em épocas secas, etc. (Azevedo et al., 2006); entretanto, a aplicação de produtos químicos continua sendo o método mais utilizado (Johnny et al., 2011). Por este motivo, os níveis de resíduos de agrotóxicos em pimentão in natura, estão atualmente entre os mais elevados 
(Agência Nacional de Vigilância Sanitária, 2013). Nesse contexto, o interesse em métodos alternativos para o controle de doenças de plantas, como o uso de extratos vegetais e seus metabólitos secundários, como agentes antimicrobianos, têm aumentado nos últimos anos (Abdel-Monaim et al., 2011).

Extratos de diversas espécies vegetais apresentam potencial contra fungos fitopatogênicos (Plodpai et al., 2013; Sukorini et al., 2013). Com relação a antracnose em pimentas (Capsicum spp.), há relatos do controle in vitro com uso de Centella asiatica (L.) Urb., Alpinia galanga Willd., Blumea balsamifera DC., Momordica charantia L., Piper betle L. (Johnny et al., 2011). Estudos sobre o controle da antracnose do pimentão com extratos vegetais ainda são raros, um exemplo é a utilização de Cymbopogon citratus (DC.) Stapf e Cymbopogon martini (Roxb.) Will Watson var. motia Burk. (Carvalho et al., 2008). Estudos fitoquímicos com espécies de Piper demonstram uma grande diversidade de metabólitos secundários, inclusive com ação antifúngica (Reigada et al., 2007; Johnny et al., 2011). Apesar disto, há poucos estudos com Piper marginatum sobre o controle de fitopatógenos.

Tem-se relato da eficiência de diferentes produtos de Azadirachta indica Juss., popularmente conhecida como nim (torta, extrato de folhas, óleo) no controle de fitopatógenos que causam doenças do sistema radicular, parte aérea e pós-colheita de plantas, como Oidium lycopersici Cooke \& Massee, Erysiphe polygoni (DC.), Colletotrichum lindemuthianum (Sacc. \& Magnus) Briosi \& Cavara (1889) (Carneiro, 2003, 2008; Rajamanickam et al., 2012).

O objetivo deste trabalho foi avaliar o efeito de extratos de Piper marginatum e Azadirachta indica sobre o fungo Colletotrichum scovillei e determinar o componente mais ativo no controle pós-colheita da antracnose em pimentão.

\section{Material e métodos}

Folhas de $P$. marginatum foram coletadas na Universidade Federal Rural de Pernambuco ( $\left.8^{\circ} 00^{\prime} 55^{\prime \prime S}, 34^{\circ} 56^{\prime} 50^{\prime \prime} \mathrm{W}\right)$, e um exemplar desta espécie encontra-se catalogado e registrado sob $\mathrm{n}^{-} \mathbf{5 7 5 . 3 5 6}$, no Herbário do Jardim Botânico, Rio de Janeiro, RJ. Folhas e sementes de $A$. indica foram cedidas pela Empresa Cruangi Neem do Brasil Ltda., Timbaúba, PE. O material vegetal foi desidratado em estufa $\left(40^{\circ} \mathrm{C}\right.$, por 72 horas) e, posteriormente, foi triturado e armazenado separadamente, em sacos de papel, nas condições do ambiente.

A extração, o fracionamento e a análise cromatográfica dos extratos foram realizados nos Laboratórios de Fitoquímica e Processos (Lafip) e na Central Analítica do Centro de Tecnologias Estratégicas do Nordeste (Cetene). O material vegetal seco e triturado de cada espécie $(600 \mathrm{~g})$ foi extraído em metanol $(\mathrm{MeOH})$, por método de extração acelerada por solvente ASE 350 (Dionex Corporation, Sunnyvale, CA, EUA), a $40^{\circ} \mathrm{C}$, e a 1700 psi, tendo-se adaptado metodologias de Reigada (2007) e Hanwen et al. (2012). Após a extração, o solvente foi removido em pressão reduzida, a $50^{\circ} \mathrm{C}$, em evaporador rotatório modelo RV10 Digital (IKA, Staufen, Alemanha).

O fracionamento do extrato metanólico, por cromatografia em coluna aberta (CC), foi realizado com uso de sílica gel 60, 70-230 mesh (Macherey-Nagel, Düren, Alemanha), como fase estacionária, e gradiente de solventes como eluentes, na seguinte ordem: hexano (HEX); HEX/acetato de etila (HEX/ EtOAc) $(1: 1 \mathrm{v} / \mathrm{v})$; acetato de etila $($ EtOAc); metanol/ acetato de etila $(\mathrm{MeOH} / \mathrm{EtOAc})(1: 1 \mathrm{v} / \mathrm{v})$; metanol $(\mathrm{MeOH})$. As frações foram coletadas separadamente, concentradas em evaporador rotatório. Posteriormente, foram pesadas e armazenadas em freezer $\left(-18^{\circ} \mathrm{C}\right)$, em frascos tipo âmbar hermeticamente fechados.

A separação dos componentes majoritários das frações obtidas acima foi realizada em cromatógrafo semipreparativo HPLC modelo EF1525, com detector UV Waters 1489 (Waters, São Paulo, SP, Brasil), com coluna C18 (21,2 x $150 \mathrm{~mm}, 5 \mu \mathrm{m})$, com fase móvel de acetonitrila (AcN) (pureza HPLC), com um fluxo de $10 \mathrm{~mL} \mathrm{~min}^{-1}$, em $10 \mathrm{~min}$, a $35^{\circ} \mathrm{C}$. No total, foram realizadas cinco corridas, com $0,5 \mathrm{~mL}$ da fração bioativa. $\mathrm{O}$ fracionamento por tempo (31 s por fração) resultou em 30 frações, das quais 10 apresentavam massa acima de 0,5 mg (frações 7, 9, 12, 13, 17, 18, 20, 21, 23, 29).

Inicialmente, avaliou-se a atividade dos extratos metanólicos de folhas e sementes de A. indica e folhas de $P$. marginatum sobre o desenvolvimento de C. scovillei in vitro, pelo teste de difusão em ágar (Duarte et al., 2003). Foram avaliadas diferentes concentrações dos extratos $(0,125,250,500,1.000$ e $2.000 \mathrm{ppm}$ ), incorporadas separadamente em meio de cultura batata-dextrose-ágar (BDA), a $45^{\circ} \mathrm{C}$, e 
vertidas em placas de Petri. Os extratos, antes de incorporados ao BDA, foram sempre filtrados em seringa com membrana de 0,45 $\mu \mathrm{m}$ PVDF Chromafil (Macherey-Nagel, Düren, Alemanha). Cada placa, com o meio de cultura acrescido dos tratamentos, recebeu um disco de BDA com $0,5 \mathrm{~cm}$ de diâmetro que continha as estruturas do patógeno (micélio). Posteriormente as placas foram mantidas em câmara BOD a $28^{ \pm} 2^{\circ} \mathrm{C}$, em fotoperíodo de 12 horas. Após 10 dias de incubação, foram aferidos os valores médios do diâmetro de crescimento micelial (CM) do patógeno (em milímetros), e as percentagens de inibição do crescimento micelial (PIC) foram calculadas por meio da fórmula PIC $=[($ diâmetro da testemunha - diâmetro do tratamento) / diâmetro da testemunha] x 100, para cada tratamento em relação à testemunha (Carvalho et al., 2013). Utilizouse o delineamento experimental inteiramente casualizado, com arranjo fatorial $3 \times 6$, sendo extratos de três espécies vegetais e seis concentrações de cada extrato, com quatro repetições. Cada repetição foi constituída por uma placa de Petri. Realizou-se a análise de regressão, e as equações foram ajustadas aos dados experimentais de cada espécie vegetal estudada. Utilizou-se o programa Assistat versão 7.6 beta (Silva \& Azevedo, 2009).

Conforme descrito anteriormente, o extrato mais ativo no controle de $C$. scovillei foi fracionado por cromatografia em coluna aberta (CC), o que resultou em quatro frações: hexano (HEX); HEX/acetato de etila (HEX/EtOAc); acetato de etila (EtOAc); metanol/ acetato de etila (MeOH/EtOAc); e metanol $(\mathrm{MeOH})$. Os efeitos destas frações foram avaliados no CM e PIC in vitro. As frações foram testadas a concentrações correspondentes ao rendimento do fracionamento (24 mg da fração HEX/EtOAc; $103 \mu \mathrm{g}$ fração EtOAc; $5 \mathrm{mg}$ fração EtOAc/MeOH; e $2 \mathrm{mg}$ da fração $\mathrm{MeOH}$ ). $\mathrm{O}$ delineamento inteiramente casualizado foi utilizado, com cinco tratamentos (frações e testemunha água destilada) e quatro repetições; as médias foram comparadas pelo teste de Tukey, a 5\% de probabilidade, com uso do programa Assistat versão 7.6 beta (Silva \& Azevedo, 2009).

As frações HEX/EtOAc, EtOAc, EtOAc/MeOH e $\mathrm{MeOH}$ de $P$. marginatum foram também avaliadas no controle de $C$. scovillei em pós-colheita. Realizaramse ferimentos em frutos de pimentão, previamente desinfestados com hipoclorito de sódio $(1,5 \%)$ por
3 min, de acordo com Menezes \& Assis (2004). As frações foram inoculadas à concentração de $50 \mu \mathrm{L}$ por fruto. Duas horas após a aplicação dos tratamentos, os frutos foram infectados com $50 \mu \mathrm{L}$ da suspensão de C. scovillei à concentração de $1 \times 10^{6}$ conídios $\mathrm{mL}^{-1}$. Como testemunha, utilizaram-se frutos tratados com água destilada esterilizada (ADE) e infectados com o fitopatógeno. Foram colocados quatro frutos por bandeja, equidistantes entre si, e cada fruto foi considerado uma repetição. As bandejas foram colocadas em sacos de plástico (câmara úmida) e mantidas à temperatura ambiente $\left(26^{\circ} \mathrm{C} \pm 2^{\circ} \mathrm{C}\right)$. A avaliação foi realizada 10 dias após a inoculação, tendo-se determinado o crescimento micelial e a percentagem de inibição do diâmetro da lesão (PIDL), com fórmula adaptada de Carvalho et al. (2013). Utilizou-se o delineamento experimental inteiramente casualizado, com cinco tratamentos (frações e testemunha) e quatro repetições. As médias foram comparadas pelo teste de Tukey, a 5\% de probabilidade, com uso do programa Assistat versão 7.6 beta (Silva \& Azevedo, 2009).

A fração mais ativa foi submetida ao segundo fracionamento biomonitorado (Koehn \& Carter, 2005) em HPLC, o que resultou em frações majoritárias. Estas frações foram utilizadas segundo a mesma metodologia dos experimentos anteriores, e as mesmas variáveis foram avaliadas (in vitro e em pós-colheita). As concentrações das frações majoritárias foram padronizadas para 1,5 ppm. O fungicida mancozeb, à concentração recomendada pelo fabricante $\left(2,5 \mathrm{mg} \mathrm{mL} \mathrm{mL}^{-1}\right)$, foi comparado às frações deste experimento. $\mathrm{O}$ delineamento experimental inteiramente casualizado foi utilizado com 14 tratamentos (água destilada como testemunha; fração EtOAc; fração acetato de etila (AcN/EtOAc); as frações 7, 9, 12, 13, 17, 18, 20, 21, 23, 29; e mancozeb), em quatro repetições. As médias foram comparadas pelo teste de Tukey, a 5\% de probabilidade, com o programa Assistat versão 7.6 beta (Silva \& Azevedo, 2009).

\section{Resultados e Discussão}

Os extratos metanólicos reduziram significativamente $(\mathrm{p} \leq 0,05)$ o crescimento micelial de $C$. scovillei (Figura 1). Apesar de os extratos de folhas e sementes de nim (A. indica) terem mostrado efeito sob as duas 
variáveis analisadas no presente estudo, o extrato de capeba (P. marginatum) mostrou-se mais eficiente.

Alguns autores relataram efeitos de extratos e componentes ativos de folhas e sementes de nim no controle de fitopatógenos. El-Kholie et al. (2012) observaram níveis significativos de inibição de CM dos fungos Fusarium oxysporum Kühn, Rhizoctonia solani Kuhn, Alternaria solani e Sclerotinia sclerotiorum, ao utilizar diferentes concentrações de nim. Ao testar o extrato de semente de nim, na inibição de doenças pós-colheita de frutas ocasionadas por Monilinia fructicola, Penicillium expansum, Alternaria alternata, Wang et al. (2010) observaram que o nim inibiu o $\mathrm{CM}$ dos patógenos avaliados in vitro e em pós-colheita.

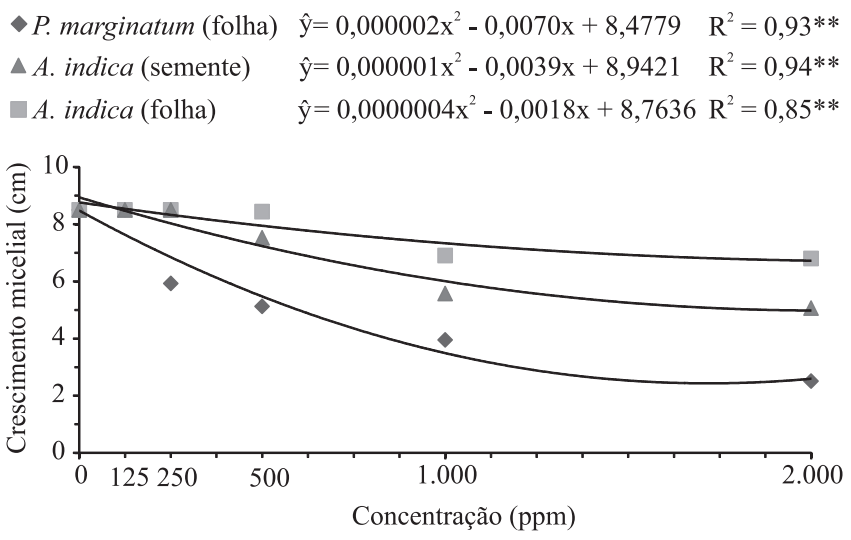

- P. marginatum (folha) $\hat{\mathrm{y}}=-0,00002 \mathrm{x}^{2}+0,0831 \mathrm{x}+0,2672 \quad \mathrm{R}^{2}=0,93 * *$ $\square$ A. indica (folha) $\hat{\mathrm{y}}=-0,000005 \mathrm{x}^{2}+0,0219 \mathrm{x}-3,1383 \mathrm{R}^{2}=0,85 * *$ $\Delta$ A. indica (semente) $\quad \hat{\mathrm{y}}=-0,00001 \mathrm{x}^{2}+0,0456 \mathrm{x}-5,175 \quad \mathrm{R}^{2}=0,94 * *$

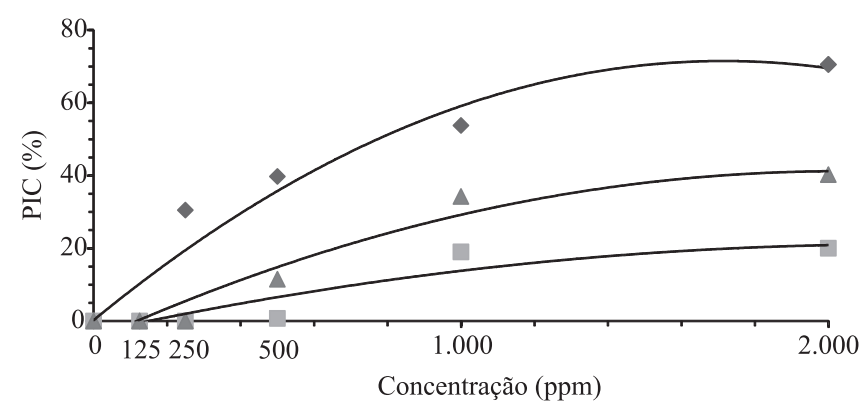

Figura 1. Relação do crescimento micelial e porcentagem de inibição do crescimento micelial (PIC) de Colletotrichum scovillei em função das concentrações de extratos metanólicos de Piper marginatum (folha), Azadirachta indica (folha) e A. indica (semente), in vitro após 10 dias de incubação.
Entretanto, assim como no presente estudo, outros autores também verificaram que o extrato de nim, quando comparado a extratos de outras espécies vegetais, não é eficiente no controle de alguns fungos fitopatogênicos. Silva et al. (2012) observaram que o extrato de nim não reduziu o $\mathrm{CM}$ de Colletotrichum gloeosporioides. Almeida et al. (2009) utilizaram extratos de várias espécies vegetais, para o controle de Colletotrichum acutatum, e verificaram que enquanto os extratos de Ruta graveolens L., Artemisia absinthium e Allium sativum apresentaram potencial fungitóxico e influenciaram o CM, a esporulação e a germinação de esporos, o extrato de nim favoreceu o crescimento micelial e promoveu alta esporulação e germinação de esporos. Prince \& Prabakaran (2011) avaliaram diferentes espécies vegetais, entre as quais $A$. indica e Piper betle (Piperaceae), contra Colletotrichum falcatum, e verificaram que o extrato de nim foi o menos eficiente no controle do fitopatógeno in vitro, enquanto $P$. betle apresentou resultados significativos de inibição do fungo. Verificamos, no presente trabalho, que o nim teve pouca influência sobre o desenvolvimento de C. scovillei, especialmente em comparação ao efeito antifúngico do extrato de $P$. marginatum, que foi promissor no controle do fitopatógeno.

Extratos de espécies do gênero Piper já foram testados contra diversos fitopatógenos in vitro: $P$. chaba Hunter inibiu o crescimento micelial de $F$. oxysporum, Phytophthora capsici Leonian., Colletotrichum capsici, F. solani (Mart.) Sacc. e R. solani (Rahman et al., 2011). Piper auritum Kunth e P. holtonii C. DC. também inibiram o crescimento micelial de C. acutatum J. H. Simmonds (1968), C. gloeosporioides (Penz.) Penz. \& Sacc. e Botryodiplodia theobromae (Pineda M. et al., 2012). Os resultados observados no presente trabalho, em que foi verificada a eficiência de Piper marginatum no controle de um importante fungo fitopatogênico do pimentão, corroboram estes estudos.

Provavelmente, alguns metabólitos secundários afetam de forma distinta os diversos fitopatógenos. Johnny et al. (2011) avaliaram diferentes espécies vegetais e verificaram que o extrato bruto de folhas de $P$. betle, a doses muito baixas $\left(0,01 \mu \mathrm{g} \mathrm{mL}^{-1}\right)$, apresentou maior atividade antifúngica na inibição do crescimento micelial de C. capsici, agente causal da antracnose do pimentão. 
$\mathrm{O}$ aumento das concentrações dos extratos promoveu diminuição de forma quadrática $(\mathrm{p} \leq 0,01)$ do crescimento micelial (Figura 1). A relação entre a percentagem de inibição do crescimento micelial e o aumento da concentração destes extratos, se ajustou a modelo quadrático, para as três espécies vegetais $(\mathrm{p} \leq 0,01)$.

O fracionamento do extrato metanólico de P. marginatum produziu $24 \mathrm{mg}$ da fração HEX/EtOAc; $103 \mu \mathrm{g}$ da fração EtOAc; $5 \mathrm{mg}$ da fração EtOAc/ $\mathrm{MeOH}$; e 2 mg da fração $\mathrm{MeOH}$ (Tabela 1). Todas as frações do extrato metanólico de $P$. marginatum controlaram o desenvolvimento de $C$. scovillei in vitro e em pós-colheita (Tabela 2). Entretanto, ressaltase que a concentração utilizada da fração EtOAc foi inferior à das demais $\left(0,036 \mathrm{mg} \mathrm{mL}^{-1}\right)$, pois as frações foram testadas a concentrações que corresponderam ao rendimento do fracionamento. Pelo fato de ter sido utilizada em menor concentração e ter obtido resultados compatíveis às frações mais concentradas, a fração EtOAc foi considerada como a de maior atividade antifúngica.

Todos as frações majoritárias, obtidas da fração EtOAc em HPLC, diferiram estatisticamente da testemunha (Tabela 2). A fração 12 foi significativamente mais ativa do que as demais, em todas as variáveis analisadas, tanto in vitro como em pós-colheita. De acordo com os resultados obtidos, $P$. marginatum apresenta compostos com potencial antifúngico. Alguns trabalhos já apresentaram relatos sobre compostos identificados e comprovados como

Tabela 1. Avaliação de frações do extrato metanólico de Piper marginatum, com o crescimento micelial $(\mathrm{CM})$ e a percentagem de inibição do crescimento micelial (PIC) de Colletotrichum scovillei in vitro e em pós-colheita ${ }^{(1)}$.

\begin{tabular}{|c|c|c|c|c|c|c|}
\hline \multirow[t]{2}{*}{ Tratamento } & \multicolumn{2}{|c|}{ Rendimento Concentração } & \multicolumn{2}{|c|}{ In vitro } & \multicolumn{2}{|c|}{ Pós-colheita } \\
\hline & $(\mathrm{mo})$ & $\left(\mathrm{mg} \mathrm{mL}^{-1}\right)$ & $\begin{array}{c}\mathrm{CM} \\
(\mathrm{cm})\end{array}$ & $\begin{array}{l}\text { PIC } \\
(\%)\end{array}$ & $\begin{array}{c}\mathrm{CM} \\
(\mathrm{cm})\end{array}$ & $\begin{array}{l}\text { PIC } \\
(\%)\end{array}$ \\
\hline Testemunha & 0,000 & 0,0000 & $8,00 \mathrm{a}$ & $0,00 \mathrm{c}$ & $3,00 \mathrm{a}$ & $0,00 \mathrm{~b}$ \\
\hline HEX/EtOAc & 24,000 & 0,5526 & $1,76 b$ & $79,26 b$ & $1,43 b$ & $52,5 \mathrm{a}$ \\
\hline EtOAc & 0,103 & 0,0360 & $1,56 \mathrm{~b}$ & $81,62 b$ & $1,24 b$ & $58,73 a$ \\
\hline EtOAc/MeOH & 5,000 & 0,2526 & $0,89 \mathrm{c}$ & $89,56 a$ & $1,73 b$ & $42,48 \mathrm{a}$ \\
\hline $\mathrm{MeOH}$ & 2,000 & 0,1612 & $0,96 \mathrm{~d}$ & $88,68 \mathrm{a}$ & $1,65 b$ & $45,00 \mathrm{a}$ \\
\hline CV (\%) & & & 3,7 & 2,723 & 21,86 & 33,12 \\
\hline
\end{tabular}

${ }^{(1)}$ Médias seguidas de letras iguais não diferem entre si, pelo teste de Tukey, a 5\% de probabilidade. HEX, hexano; EtOAc, acetato de etila; $\mathrm{MeOH}$, metanol. Testemunha, água destilada. antimicrobianos em espécies do gênero Piper (Rahman \& Kang, 2011).

O fungicida mancozeb à dose recomendada pelo fabricante $\left(2,5 \mathrm{mg} \mathrm{mL}^{-1}\right)$ não reduziu totalmente $\mathrm{o}$ crescimento micelial de $C$. scovillei in vitro e em pós-colheita. Johnny et al. (2011) compararam o extrato bruto de $P$. betle a um fungicida comercial e verificaram que o fungicida (Kocide 101) apresentou $87,24 \%$ e $P$. betle $85 \%$ de inibição do crescimento de C. capsici, que são valores muito próximos entre si, o que indica que o extrato bruto de $P$. betle é promissor no controle alternativo deste fitopatógeno. Enquanto Johnny et al. (2011) observaram resultados satisfatórios para o extrato bruto de $P$. betle, no presente trabalho, obtivemos fração majoritária que é promissora como fonte para a produção de um antifúngico natural. A continuidade dos estudos, por meio da elucidação estrutural destas frações majoritárias, em especial a fração 12 , possibilitará o desenvolvimento de futuras formulações para o controle de C. scovillei e testes em outros fitopatógenos.

Tabela 2. Avaliação das frações majoritárias do extrato metanólico de Piper marginatum, com o crescimento micelial $(\mathrm{CM})$, percentagem de inibição do crescimento micelial (PIC) e percentagem de inibição do diâmetro da lesão (PIDL) de Colletotrichum scovillei in vitro e em pós-colheita, em comparação ao fungicida mancozeb ${ }^{(1)}$.

\begin{tabular}{|c|c|c|c|c|c|}
\hline \multirow[t]{2}{*}{ Tratamento } & \multirow{2}{*}{$\begin{array}{l}\text { Concentração } \\
\left(\mu \mathrm{g} \mathrm{mL}^{-1}\right)\end{array}$} & \multicolumn{2}{|c|}{ In vitro } & \multicolumn{2}{|c|}{ Pós-colheita } \\
\hline & & $\mathrm{CM}(\mathrm{cm})$ & PIC (\%) & $\mathrm{CM}(\mathrm{cm})$ & PIDL (\%) \\
\hline Testemunha & 0,0 & $8,50 \mathrm{a}$ & 0,00 & $4,50 \mathrm{a}$ & 0,00 \\
\hline Fração EtOAc & 1,5 & $8,50 \mathrm{a}$ & 0,00 & $4,50 \mathrm{a}$ & 0,00 \\
\hline Fração AcN/EtOAc & 1,5 & $3,33 \mathrm{e}$ & $60,88 \mathrm{c}$ & $1,53 \mathrm{ef}$ & $59,5 \mathrm{bc}$ \\
\hline Fração 7 & 1,5 & $3,76 \mathrm{~d}$ & $55,81 \mathrm{~cd}$ & $1,75 \mathrm{de}$ & $55,00 \mathrm{~cd}$ \\
\hline Fração 9 & 1,5 & $4,45 \mathrm{c}$ & $47,65 \mathrm{ef}$ & $2,35 \mathrm{c}$ & $43,00 \mathrm{e}$ \\
\hline Fração 12 & 1,5 & $1,50 \mathrm{~g}$ & $82,43 a$ & $0,44 \mathrm{~g}$ & $81,25 \mathrm{a}$ \\
\hline Fração 13 & 1,5 & $4,55 \mathrm{c}$ & $46,47 f$ & $2,38 \mathrm{c}$ & $42,50 \mathrm{e}$ \\
\hline Fração 17 & 1,5 & $4,55 b$ & $46,47 f$ & $2,25 \mathrm{c}$ & $45,00 \mathrm{e}$ \\
\hline Fração 18 & 1,5 & $4,36 \mathrm{~d}$ & $48,75 \mathrm{ef}$ & $2,19 \mathrm{c}$ & $46,25 \mathrm{e}$ \\
\hline Fração 20 & 1,5 & $4,63 \mathrm{c}$ & $45,51 \mathrm{f}$ & $2,24 \mathrm{c}$ & $45,25 \mathrm{e}$ \\
\hline Fração 21 & 1,5 & $4,03 \mathrm{c}$ & $52,65 \mathrm{de}$ & $1,90 \mathrm{~d}$ & $52,00 \mathrm{~d}$ \\
\hline Fração 23 & 1,5 & $4,39 \mathrm{c}$ & $48,38 \mathrm{ef}$ & $2,16 \mathrm{c}$ & $46,75 \mathrm{e}$ \\
\hline Fração 29 & 1,5 & $4,76 b$ & $44,05 f$ & $2,31 \mathrm{c}$ & $43,75 \mathrm{e}$ \\
\hline Fungicida mancozeb & 2,5 & $2,59 \mathrm{f}$ & $69,56 \mathrm{~b}$ & $1,38 \mathrm{f}$ & $62,50 \mathrm{~b}$ \\
\hline $\mathrm{CV}(\%)$ & & 5,39 & 7,08 & 4,11 & 4,20 \\
\hline
\end{tabular}

${ }^{(1)}$ Médias seguidas de letras iguais, nas colunas, não diferem entre si, pelo teste de Tukey, a 5\% de probabilidade. EtOAc, acetato de etila; AcN, acetonitrila. Testemunha, água destilada. 


\section{Conclusões}

1. Os extratos metanólicos de folhas de Piper marginatum a $250 \mathrm{ppm}$, de folhas de Azadirachta indica a 1.000 e 2.000 ppm e de sementes de $A$. indica a $500 \mathrm{ppm}$ inibem o crescimento micelial de Colletotrichum scovillei in vitro.

2. A fração acetato de etila do extrato metanólico de $P$. marginatum reduz significativamente o crescimento in vitro de $C$. scovillei.

3. Uma fração purificada da fração acetato de etila do extrato metanólico de $P$. marginatum reduz o crescimento de C. scovillei, tanto in vitro como em pós-colheita, de modo mais eficiente do que o fungicida mancozeb.

\section{Agradecimentos}

Ao Conselho Nacional de Desenvolvimento Científico e Tecnológico $(\mathrm{CNPq})$, por concessão da bolsa de estudo; à Dra. Elsie Guimaraes, pela disponibilidade em confirmar a identificação botânica da espécie vegetal utilizada no presente estudo; ao Centro de Tecnologias Estratégicas do Nordeste (Cetene), pelo espaço cedido à pesquisa.

\section{Referências}

ABDEL-MONAIM, M.F.; ABO-ELYOUSR, K.A.M.; MORSY, K.M. Effectiveness of plant extracts on suppression of damping-off and wilt diseases of lupine (Lupinus termis Forsik). Crop Protection, v.30, p.185-191, 2011. DOI: 10.1016/j.cropro.2010.09.016.

AGÊNCIA NACIONAL DE VIGILÂNCIA SANITÁRIA. Programa de análise de resíduos de agrotóxicos em alimentos (Para): relatório de atividades de 2011 e 2012. Brasília: Anvisa, 2013. Disponível em: <http://portal.anvisa.gov.br/wps/ wcm/connect/d480f50041 ebb7a09db8bd3e2b7e7e4d/Relat $\%$ C3\%B3rio\%2BPARA\%2B2011-12\%2B-\%2B30 $10 \quad 13 \quad 1$. pdf?MOD=AJPERES $>$. Acesso em: 14 fev. 2014.

ALMEIDA, T.F.; CAMARGO, M.; PANIZZI, R. de C. Efeito de extratos de plantas medicinais no controle de Colletotrichum acutatum, agente causal da flor preta do morangueiro. Summa Phytopathologica, v.35, p.196-201, 2009. DOI: 10.1590/ S0100-54052009000300006.

AZEVEDO, C.P. de; CAFÉ FILHO, A.C.; HENZ, G.P.; REIS, A. Recomendações de manejo da antracnose do pimentão e das pimentas. Brasília: Embrapa Hortaliças, 2006. 4p. (Embrapa Hortaliças. Comunicado técnico 35).

CARNEIRO, S.M. de T.P.G. Efeito de extratos de folhas e do óleo de nim sobre o oídio do tomateiro. Summa Phytopathologica, v.29, p.262-265, 2003.
CARNEIRO, S.M. de T.P.G. Efeito do Nim (Azadirachta indica) sobre oídio e antracnose. Londrina: Instituto Agronômico do Paraná, 2008. 15p. (Instituto Agronômico do Paraná. Informe da pesquisa, 155).

CARVALHO, J.B.; SCHWAN-ESTRADA, K.R.F.; BONALDO, S.M.; CRUZ, M.E.S.; CARLOS, M.M.; STANGARLIN, J.R. Fungitoxicidade de Cymbopogon citratus e Cymbopogon martinii a Colletotrichum gloeosporioides em frutos de pimentão. Revista Brasileira de Plantas Medicinais, v.10, p.88-93, 2008.

CARVALHO, R.R. da C. e; LARANJEIRA, D.; CARVALHO FILHO, J.L.S. de; SOUZA, P.E. de; BLANK, A.F.; ALVES, P.B.; JESUS, H.C.R. de; WARWICK, D.R.N. In vitro activity of essential oils of Lippia sidoides and Lippia gracilis and their major chemical components against Thielaviopsis paradoxa, causal agent of stem bleeding in coconut palms. Química Nova, v.36, p.241-244, 2013. DOI: $10.1590 / \mathrm{S} 0100-40422013000200007$.

DAMM, U.; CANNON, P.F.; WOUDENBERG, J.H.C.; CROUS, P.W. The Colletotrichum acutatum species complex. Studies in Mycology, v.73, p.37-113, 2012. DOI: 10.3114/sim0010.

DUARTE, S.; KOO, H.; BOWEN, W.H.; HAYACIBARA, M.F.; CURY, J.A.; IKEGAKI, M.; ROSALEN, P.L. Effect of a novel type of propolis and its chemical fractions on glucosyltransferases and on growth and adherence of mutans Streptococci. Biological and Pharmaceutical Bulletin, v.26, p.527-531, 2003. DOI: 10.1248/ bpb.26.527.

EL-KHOLIE, E.M.; ABDELREHEEM, M.A.T.; KHADER, S.A. Azadirachta indica extracts influenced some pathogenic fungi. African Journal of Microbiology Research, v.6, p.5645-5649, 2012.

HANWEN, S.; XUSHENG, G.; YUNKAI, L.; ANBANG, W. Application of accelerated solvent extraction in the analysis of organic contaminants, bioactive and nutritional compounds in food and feed. Journal of Chromatography A, v.1237, p.1-23, 2012. DOI: 10.1016/j.chroma.2012.03.003.

JOHNNY, L.; YUSUF, U.K.; NULIT, R. Antifungal activity of selected plant leaves crude extracts against a pepper anthracnose fungus, Colletotrichum capsici (Sydow) butler and bisby (Ascomycota: Phyllachorales). African Journal of Biotechnology, v.10, p.4157-4165, 2011.

KIM, H.J.; LEE, E.J.; PARK, S.H.; LEE, H.-S.; CHUNG, N. Biological control of anthracnose (Colletotrichum gloeosporioides) in pepper and cherry tomato by Streptomyces sp. A1022. Journal of Agricultural Science, v.6, p.54-62, 2014.

KOEHN, F.E.; CARTER, G.T. The evolving role of natural products in drug discovery. Nature Reviews Drug Discovery, v.4, p.206-220, 2005. DOI: 10.1038/nrd1657.

MENEZES, M.; ASSIS, S.M.P. Guia prático para fungos fitopatogênicos. Recife: Ed. da UFRPE, 2004. 183p.

PINEDA M., R.; VIZCAÍNO P., S.; GARCÍA P., C.M.; GIL G., J.H.; DURANGO R., D.L. Chemical composition and antifungal activity of Piper auritum Kunth and Piper holtonii C. DC. against phytopathogenic fungi. Chilean Journal of Agricultural Research, v.72, p.507-515, 2012. DOI: 10.4067/ S0718-58392012000400008. 
PLODPAI, P.; PETCHARAT, V.; CHUENCHIT, S.; CHAKTHONG, S.; JOYCHARAT, N.; VORAVUTHIKUNCHAI, S.P. Desmos chinensis: a new candidate as natural antifungicide to control rice diseases. Industrial Crops and Products, v.42, p.324-331, 2013. DOI: 10.1016/j.indcrop.2012.05.038.

PRINCE, L.; PRABAKARAN, P. Antifungal activity of medicinal plants against plant pathogenic fungus Colletotrichum falcatum. Asian Journal of Plant Science and Research, v.1, p.84-87, 2011.

RAHMAN, A.; AL-REZA, S.M.; KANG, S.C. Antifungal activity of essential oil and extracts of Piper chaba Hunter against phytopathogenic fungi. Journal of the American Oil Chemists' Society, v.88, p.573-579, 2011.

RAJAMANICKAM, S.; SETHURAMAN, K.; SADASAKTHI, A. Exploitation of phytochemicals from plants extracts and its effect on growth of Colletotrichum capsici (Syd.) Butler and Bisby causing anthracnose of chilli (Capsicum annuum L.). Plant Pathology Journal, v.11, p.87-92, 2012. DOI: 10.3923/ ppj.2012.87.92.

REIGADA, J.B.; TCACENCO, C.M.; ANDRADE, L.H.; KATO, M.J.; PORTO, A.L.M; LAGO, J.H.G. Chemical constituents from Piper marginatum Jacq. (Piperaceae) - antifungal activities and kinetic resolution of (RS)-marginatumol by Candida antarctica lipase (Novozym 435). Tetrahedron: Asymmetry, v.18, p.1054-1058, 2007. DOI: 10.1016/j.tetasy.2007.05.006.

SILVA, F. de A.S. e; AZEVEDO, C.A.V. de. Principal components analysis in the software Assistat-statistical assistance. In: WORLD CONGRESS ON COMPUTERS IN AGRICUlTURE, 7, 2009, Reno. Proceedings. Reno: American Society of Agricultural and Biological Engineers, 2009. DOI: $10.13031 / 2013.29066$.

SILVA, J.L. da; TEIXEIRA, R.N.V.; SANTOS, D.I.P.; PESSOA, J.O. Atividade antifúngica de extratos vegetais sobre o crescimento in vitro de fitopatógenos. Revista Verde, v.7, p.80-86, 2012.

SUKORINI, H.; SANGCHOTE, S.; KHEWKHOM, N. Control of postharvest green mold of citrus fruit with yeasts, medicinal plants, and their combination. Postharvest Biology and Technology, v.79, p.24-31, 2013. DOI: 10.1016/j. postharvbio.2013.01.001.

WANG, J.; CAO, J.L.J.; JIANG, W. Antifungal activities of neem (Azadirachta indica) seed kernel extracts on postharvest diseases in fruits. African Journal of Microbiology Research, v.4, p.1100-1104, 2010.

Recebido em 22 de outubro de 2013 e aprovado em 30 de janeiro de 2014 